\title{
Dietary food patterns and glucose/insulin homeostasis: a cross-sectional study involving 24,182 adult Americans
}

\author{
Mohsen Mazidi ${ }^{1,2^{*}}$, Andre Pascal Kengne ${ }^{3}$, Dimitri P. Mikhailidis ${ }^{4}$, Peter P. Toth ${ }^{5,6}$, Kausik K. Ray ${ }^{7}$ \\ and Maciej Banach ${ }^{8,9,10}$
}

\begin{abstract}
Aim: To investigate the association of major dietary patterns with glucose and insulin homeostasis parameters in a large American sample. The association between dietary patterns (DP) derived via principal components analysis (PCA), with glucose/insulin homeostasis parameters was assessed. The likelihood of insulin resistance (IR) across the DPs quarters was also explored.

Method: The United States National Health and Nutrition Examination Survey (NHANES) participants during 2005-2012 were included if they underwent measurement of dietary intake as well as glucose and insulin homeostasis parameters. Analysis of covariance (ANCOVA) and adjusted logistic and linear regression models were employed to account for the complex survey design and sample weights.

Results: A total of 24,182 participants were included; $48.9 \%(n=11,815)$ were men. Applying PCA revealed three DP (56. $8 \%$ of variance): the first was comprised mainly of saturated fat (SFA), total fat, mono-unsaturated fatty acids (MUFA) and carbohydrate (CHO); the second is highly enriched with vitamins, trace elements and dietary fiber; and the third was composed of polyunsaturated fatty acids (PUFA), cholesterol and protein. Among the total population, after adjustment for age, sex, race, C-reactive protein, smoking, and physical activity, glucose homeostasis factors, visceral adiposity index and lipid accumulation product improved across the quarters of the first and third DP; and a reverse pattern with the second DP. The same trend was observed for the non-diabetic subjects. Moreover, subjects with higher adherence to the first and third DP had higher likelihood for developing IR, whereas there was a lower likelihood for the second DP.

Conclusion: This study shows that the DP heavily loaded with CHO, SFA, PUFA, protein, total fat and MUFA as well as high-cholesterol-load foods is associated with impaired glucose tolerance; in contrast, the healthy pattern which is high in vitamins, minerals and fiber may have favourable effects on insulin sensitivity and glucose tolerance.
\end{abstract}

Keywords: Dietary patterns, Insulin resistance, Glucose homeostasis, Insulin homeostasis

\section{Background}

$\beta$-Cell dysfunction and hyperinsulinaemia are involved in the development of diabetes mellitus (DM) [1-3]. The triglycerides/glucose index (TyG), a product of triglycerides (TG) and fasting blood glucose (FBG), has also been used as an alternative marker of IR among adults $[4,5]$.

\footnotetext{
* Correspondence: moshen@genetics.ac.cn

${ }^{1}$ Key State Laboratory of Molecular Developmental Biology, Institute of Genetics and Developmental Biology, Chinese Academy of Sciences, Chaoyang, Beijing, China

${ }^{2}$ Institute of Genetics and Developmental Biology, College, University of Chinese Academy of Science, Beijing, China

Full list of author information is available at the end of the article
}

Recent investigation has reported that the TyG index could be a better alternative marker of IR among adolescents compared with the homeostatic model assessment-insulin resistance (HOMA-IR) [5]. There are complex interactions between social, behavioural, cultural, physiological, metabolic and genetic factors that are involved in the development of dysglycaemia and hyperinsulinaemia. It is widely accepted that dietary factors are important in preventing impaired glucose metabolism [6, 7]. Relatively limited nutritional epidemiological research has been conducted to examine long-term effects of diet on glucose and insulin 
homeostasis, and previous studies have focused mainly on the risk of DM.

Studies on the impact of diet on health have traditionally focused on single nutrients or food/food groups. These approaches, however, have methodological and conceptual limitations [8-10], in the sense that they can only capture the effects of a single nutrient or food group, but not the interactions among nutrients and foods $[10,11]$. Dietary pattern (DP) analysis is an alternative approach in nutritional epidemiology $[8,12,13]$. In this approach, statistical methods are used to examine the pattern of intake of multiple foods or nutrients and derive single-exposure variables, or DPs [9]. Using the DP approach could facilitate the development of public health recommendations that are more convenient to follow [14].

Although the relationship between glucose tolerance abnormalities and dietary factors in terms of dietary intake and food patterns has been investigated, the conclusions are still contradictory [15-17]. These findings may be explained by various methodologies, such as the different techniques used for measuring diet, food patterns, or blood glucose; study design; or study population. The aim of the current study was to examine the association between DP and glucose and insulin homeostasis in a cross section of American adults.

\section{Methods}

\section{Population}

Data for participants who took part in the Nutrition and Health Examination Surveys (NHANES) from 2005 to 2012 were used [18], and were restricted to those aged $\geq 18$ years. NHANES, which has been extensively described, is an ongoing cycle of cross-sectional surveys conducted by the US National Centre for Health Statistics (NCHS) [18]. Briefly, in NHANES, complex, multistage, probability sampling procedures are used to ensure selection of participants from various geographical locations and racial/ethnic populations [18, 19]. Trained interviewers collected participants' demographic, socioeconomic, dietary, and health-related information using questionnaires administered during home visits, whereas clinical examination and dietary assessment are conducted by skilled personnel using a mobile examination centre (MEC) [18]. Further details on protocols for biochemical analyses are available elsewhere; all methods were carried out in accordance with relevant guidelines and regulations [18]. All experimental protocols were approved by the National Centre for Health Statistics [18]. Informed consent was obtained from all adult participants, and the National Centre for Health Statistics Research Ethics Review Board approved the protocol. A blood specimen was drawn from the participant's antecubital vein by a trained phlebotomist according to a standardized protocol. Fasting glucose was measured in plasma by a hexokinase method using a Roche/Hitachi 911 Analyser and Roche Modular P Chemistry Analyser. Details on high sensitivity C-reactive protein (hsCRP) concentrations measurement are available elsewhere [20]. Insulin was measured using an ELISA immunoassay (Mercodia, Uppsala, Sweden) [21]. Further details on protocols for biochemical analyses are available elsewhere [20-23].

Homeostatic model assessment of insulin resistance (HOMA-IR), $\beta$-cell function (HOMA-B) and insulin sensitivity (HOMA-IS) were calculated as follows: the homeostatic model of insulin resistance (HOMA-IR) $=$ [glucose $(\mathrm{nmol} / \mathrm{L}) *$ insulin $(\mathrm{mU} / \mathrm{mL}) / 22.5]$ using fasting values, HOMA-B $=[20 \times$ fasting insulin $(\mu \mathrm{U} / \mathrm{ml})] /$ [fasting glucose $(\mathrm{mmol} / \mathrm{l})-3 \cdot 5]$, and HOMA-S = 1/HOMA-IR $\times 100$ [24]. These indices have been developed as validated alternative tools for assessment of insulin homeostasis in epidemiological studies. The triglyceride-glucose (TyG) index was calculated as the $\ln [$ fasting triglyceride $(\mathrm{TG})(\mathrm{mg} / \mathrm{dl}) \times$ glucose $(\mathrm{mg} / \mathrm{dl}) / 2$ ] [25]. DM was diagnosed by self-reported history of DM or fasting plasma glucose (FBG) $\geq 126 \mathrm{mg} / \mathrm{dl}$ [26]; HOMA-IR > 2.5 indicates insulin resistance [27]. The anthropometrically predicted visceral adipose tissue (apVAT) was predicted with sex-specific validated equations that included age, BMI, and circumferences of the waist and thigh [28]. The formula for men was: 6 "waist circumference -4.41 * proximal thigh circumference +1.19 * age -213.65 ; and the formula for women was: $2.15 *$ waist circumference $-3.63 *$ proximal thigh $+1.46 *$ age $+6: 22 *$ BMI -92.713 [28]. Visceral adiposity index (VAI) was calculated using sex-specific formulas: males [WC/ $39.68+(1.88 \times$ BMI $)] \times($ TGs $/ 1.03) \times(1.31 /$ HDL $)$; Females: $[\mathrm{WC} / 36.58+(1.89 \times \mathrm{BMI})] \times(\mathrm{TGs} / 0.81) \times(1.52 / \mathrm{HDL})$, where both TGs and high density lipoprotein (HDL) levels are expressed in mmol/L [29]. Lipid accumulation product (LAP) index, a novel measure of central lipid accumulation and predictor of metabolic syndrome and cardiovascular disease, was calculated as [WC $(\mathrm{cm})-65] \times$ [triglycerides $(\mathrm{mmol} / \mathrm{L})]$ in men, and [WC $(\mathrm{cm})-58] \times$ [triglycerides $(\mathrm{mmol} / \mathrm{L})]$ in women [30]. Smoking status was selfreported.

\section{Statistical analysis}

Statistical analyses used the complex sample module of the SPSS $^{\oplus}$ software version 22.0 (IBM Corp, Armonk, NY), applying the Centers for Disease Control (CDC) guidelines for complex NHANES data analysis, accounting for the masked variance and recommended weighting scheme [31]. The principal component analysis (PCA) was applied to determine the dietary patterns as previously described [32]. Briefly, orthogonal transformation (varimax procedure) was applied to derive nutrient patterns based on the nutrient compounds. Factors were retained for further analysis based on their natural interpretation and eigenvalues on the Scree test [32, 33]. 
Factor score for each dietary pattern was calculated by summing intakes of nutrients weighted by their factor loadings [32, 33]. Adjusted (age-, sex-, race-, C-reactive protein, smoking, physical activity) mean of glucose and insulin homeostasis factors across the quarters of each food pattern, was calculated. Adjusted (age-, sex-, race-, C-reactive protein, smoking, physical activity) logistic regressions were applied to determine risk of insulin resistance across the quarters of each dietary pattern. A two-sided $p<0.05$ was used to identify statistically significant results.

\section{Results}

A total of 24,182 participants met the criteria for inclusion in the current analyses. Their characteristics are summarised in Table 1; 11,815 (48.9\%) participants were men and 12,367 (51.1\%) were women; their mean age was 45.9 years. Non-Hispanic white (68.5\%) was the largest racial group and other Hispanic (5.0\%) the smallest racial group. Furthermore, $50.2 \%$ of the participants were married, while $28.8 \%$ had achieved more education than high school (Table 1). As seen from Table 1, mean and standard error mean (SEM) of FBG and plasma insulin were $98.27 \pm 0.12(\mathrm{mg} / \mathrm{dl})$ and $12.92 \pm 0.15(\mu \mathrm{U} / \mathrm{mL})$, respectively. Moreover, when participants were divided based on their DM status, it was found that in the DM group, men were the majority (53.1\%) while in the non-DM group women $(51.6 \%)$ were more common $(p<0.001)$. DM subjects were older than the non-DM subjects (59.6 vs. 46.1 years). DM subjects had significant higher level of LAP, VAI, TyG index, Serum TG/HDL ratio and apVAT (all $\mathrm{p}<0.001$ ).

Applying PCA resulted in three dietary patterns which together explained $56.8 \%$ of variance in dietary nutrient consumption. The first dietary pattern (22.6\%) consisted of saturated fat (SFA), total fat, mono-unsaturated fatty acids (MUFA) and carbohydrate; the second dietary pattern $(18.9 \%)$ was enriched with vitamins, trace elements and dietary fiber; and the third dietary pattern (15.3\%) was enriched with polyunsaturated fatty acids (PUFA), cholesterol and protein.

The age-, sex-, race-, hsCRP, smoking, and physical activity adjusted mean of glucose homeostatic factors across the quarters of dietary patterns are shown in Table 2. Across quarters of the first dietary pattern, mean levels of markers of glucose and insulin homeostasis increased for plasma insulin (12.3 vs $15.5 \mu \mathrm{U} / \mathrm{mL}$ ), HbA1c (5.6 vs. 5.8\%), HOMA-IR (3.3 vs.4.1), HOMA-B (143.5 vs. 164.7), VAI (2.2 vs. 2.7), LAP (63.2 vs.73.8) and decreased for HOMA-S (0.58 vs. 0.49 ) (all $p<0.001$ ). The pattern was reversed across quarters of the second dietary pattern, as FBG (102.2 vs. $99.1 \mathrm{mg} / \mathrm{dl})$, plasma insulin (14.5 vs. 12.7 $\mu \mathrm{U} / \mathrm{mL}$ ), HbA1c (5.8 vs. 5.4\%), 2 h-glucose (123.1 vs.115.4 mg/dl), HOMA-IR (3.9 vs. 3.3), VAI (2.6 vs. 2.1 ),
LAP (70.6 vs. 62.8$)$ decreased while HOMA-S (0.50 vs. 0.58 ) increased (all $p<0.001$ ). The profiles of indicators of glucose and insulin homeostasis across quarters of the third DP were similar to those across quarters of the first DP, although changes in HOMA-B, HOMA-S and VAI were not significant (Table 2). When analysis was restricted to participants without known DM (91.4\% of the total sample), the results remained similar to those observed in the total sample, with only few exceptions. These exceptions related to mean $\mathrm{HbA}_{1 \mathrm{c}}$ across quarters of the first and third DP, and fasting glucose across quarters of the third DP, which were no longer significant (Table 2).

In age-, sex-, race-, hsCRP, smoking, and physical activity adjusted logistic regression, the likelihood of insulin resistance increased across quarters of the first and third DP, but decreased across quarters of the second DP. For example, relative to the lowest quarters, the odds ratio (95\% confidence interval) for prevalent insulin resistance was $1.14(1.00-1.31, p<0.001)$ for the second quarter, $1.37(1.14-1.63, \mathrm{p}<0.001)$ for the third quarter and 1.50 $(1.26-1.79, \mathrm{p}<0.001)$ for the fourth quarter of the first DP. Equivalent figures were $0.99(0.84-1.16, p=0.223), 0.88$ $(0.76-1.01, p=0.125)$ and $0.69(0.60-0.80, \mathrm{p}<0.001)$ across quarters of the second DP, and $1.03(0.89-1.20, p=0.423)$, $1.25(1.08-1.45, \mathrm{p}<0.001)$ and $1.33(1.18-1.51, \mathrm{p}<0.001)$ across quarters of the third DP.

\section{Discussion}

The results of this study reveal that subjects who consumed higher fat, PUFA, protein, carbohydrate and cholesterol had less favourable profiles of glucose and insulin homeostasis, while subjects with higher intake of vitamins, trace elements and dietary fiber had favourable profiles.

In accordance with findings from the current study, other investigators have reported that a diet rich in fruits, vegetables, and whole grains prevents or controls insulin resistance related states including impaired fasting glucose (IFG) and impaired glucose tolerance (IGT) [6]; However, consistent consumption of refined grains, high-fat dairy and sugar sweetened beverages, and a diet rich in saturated fatty acids and cholesterol increases the risk of impaired glucose and insulin regulation [34].

An association between green leafy vegetables (which are enriched with vitamins, trace elements and soluble dietary fiber) with a reduced risk of type 2 diabetes has also been reported [35, 17]. Furthermore, the Nurses' Health Study showed that a dietary pattern high in refined carbohydrate and sugar such as sugar-sweetened soft drinks, refined grains, soft drinks and processed meat, but low in cruciferous vegetables and yellow vegetables, correlated with augmented risk for type $2 \mathrm{DM}$ [36]. The Mediterranean or DASH (Dietary Approaches to Stop Hypertension) diets exert a protective effect 
Table 1 Demographic characteristics of subjects

\begin{tabular}{|c|c|c|c|c|c|}
\hline \multicolumn{2}{|l|}{ Characteristics } & \multirow{2}{*}{$\begin{array}{l}\text { Overall } \\
48.9\end{array}$} & \multirow{2}{*}{$\begin{array}{l}\text { Non-diabetic subjects } \\
48.4\end{array}$} & \multirow{2}{*}{$\begin{array}{l}\text { Diabetic subjects } \\
53.1\end{array}$} & \multirow{2}{*}{$\frac{p \text {-value }}{<0.001}$} \\
\hline Sex & Men (\%) & & & & \\
\hline & Women (\%) & 51.1 & 51.6 & 46.9 & \\
\hline Age (Years) & & $45.9 \pm 0.2$ & $46.1 \pm 0.2$ & $59.6 \pm 0.3$ & $<0.001$ \\
\hline \multirow[t]{5}{*}{ Race/Ethnicity } & White (non-Hispanic) (\%) & 68.5 & 48.3 & 42.3 & $<0.001$ \\
\hline & Non-Hispanic Black (\%) & 11.6 & 19.7 & 21.4 & \\
\hline & Mexican-American (\%) & 8.3 & 18.9 & 22.7 & \\
\hline & Other Hispanic (\%) & 5.0 & 8.5 & 8.5 & \\
\hline & Other (\%) & 6.6 & 4.6 & 5.0 & \\
\hline \multirow[t]{4}{*}{ Marital Status } & Married (\%) & 50.2 & 51.4 & 55.4 & $<0.001$ \\
\hline & Widowed (\%) & 8.8 & 7.5 & 15.1 & \\
\hline & Divorced (\%) & 10.2 & 9.9 & 12.3 & \\
\hline & Never married (\%) & 18.9 & 19.7 & 9.2 & \\
\hline \multirow[t]{3}{*}{ Education Status } & Less than high school (\%) & 12.2 & 11.7 & 21.4 & $<0.001$ \\
\hline & Completed high school (\%) & 39.0 & 39.8 & 41.8 & \\
\hline & More than high school (\%) & 48.8 & 48.5 & 36.7 & \\
\hline \multicolumn{2}{|c|}{ Body mass index $\left(\mathrm{kg} / \mathrm{m}^{2}\right)$} & $28.5 \pm 0.1$ & $28.4 \pm 0.1$ & $31.8 \pm 0.2$ & $<0.001$ \\
\hline \multicolumn{2}{|c|}{ Waist circumference $(\mathrm{cm})$} & $98 \pm 0.3$ & $97 \pm 1$ & $107 \pm 1$ & $<0.001$ \\
\hline \multicolumn{2}{|c|}{$\begin{array}{l}\text { Anthropometrically predicted } \\
\text { visceral adipose tissue }\end{array}$} & $179.9 \pm 1.8$ & $174.8 \pm 2.3$ & $249.2 \pm 1.9$ & $<0.001$ \\
\hline \multicolumn{2}{|c|}{ Serum triglycerides (mg/dl) } & $154 \pm 3$ & $147 \pm 3$ & $215 \pm 4$ & $<0.001$ \\
\hline \multicolumn{2}{|c|}{ Serum total cholesterol (mg/dl) } & $196 \pm 1$ & $190 \pm 3$ & $195 \pm 2$ & $<0.001$ \\
\hline \multicolumn{2}{|c|}{ Serum high density lipoprotein (mg/dl) } & $53 \pm 1.6$ & $53 \pm 2$ & $47.1 \pm 1$ & $<0.001$ \\
\hline \multicolumn{2}{|c|}{ Serum TG/HDL ratio } & $3.5 \pm 0.1$ & $3.3 \pm 0.1$ & $5.5 \pm 0.7$ & $<0.001$ \\
\hline \multicolumn{2}{|c|}{ Serum hsCRP (mg/dl) } & $0.39 \pm 0.05$ & $0.40 \pm 0.06$ & $0.63 \pm 0.02$ & $<0.001$ \\
\hline \multicolumn{2}{|c|}{ Serum Apolipoprotein (B) (mg/dL) } & $93 \pm 2$ & $95 \pm 3$ & $97 \pm 2$ & $<0.001$ \\
\hline \multicolumn{2}{|c|}{ Systolic blood pressure (mmHg) } & $121 \pm 1$ & $122 \pm 0.3$ & $132 \pm 1$ & $<0.001$ \\
\hline \multicolumn{2}{|c|}{ Diastolic blood pressure (mmHg) } & $70 \pm 1$ & $68 \pm 0.4$ & $70 \pm 0.3$ & $<0.001$ \\
\hline \multicolumn{2}{|c|}{ Fasting blood glucose (mg/dl) } & $98 \pm 0.1$ & $91 \pm 0.4$ & $182 \pm 1$ & $<0.001$ \\
\hline \multicolumn{2}{|c|}{ Plasma Insulin $(\mu \mathrm{U} / \mathrm{mL})$} & $12.9 \pm 0.1$ & $12.7 \pm 0.4$ & $22.3 \pm 0.1$ & $<0.001$ \\
\hline \multicolumn{2}{|l|}{ HOMA-IR } & $3.3 \pm 0.08$ & $2.9 \pm 0.08$ & $9.6 \pm 0.06$ & $<0.001$ \\
\hline \multicolumn{2}{|l|}{ HOMA-B } & $15.6 \pm 2.5$ & $162.4 \pm 3.6$ & $85.5 \pm 1.2$ & $<0.001$ \\
\hline \multicolumn{2}{|l|}{ HOMA-S } & $0.56 \pm 0.01$ & $0.60 \pm 0.02$ & $0.25 \pm 0.01$ & $<0.001$ \\
\hline \multicolumn{2}{|l|}{$\mathrm{HbA1c}(\%)$} & $5.5 \pm 0.01$ & $5.4 \pm 0.01$ & $7.4 \pm 0.02$ & $<0.001$ \\
\hline \multicolumn{2}{|c|}{ 2-h blood glucose (mg/dL) } & $116 \pm 1$ & $117 \pm 1$ & $273 \pm 0.3$ & $<0.001$ \\
\hline \multicolumn{2}{|l|}{ TyG index } & $8226 \pm 72$ & $6785 \pm 83$ & $20,590 \pm 234$ & $<0.001$ \\
\hline \multicolumn{2}{|c|}{ Lipid Accumulation Product } & $68 \pm 0.5$ & $63 \pm 0.2$ & $112 \pm 0.9$ & $<0.001$ \\
\hline Visceral Adiposity & & $2.5 \pm 0.02$ & $2.3 \pm 0.03$ & $4.0 \pm 0.05$ & $<0.001$ \\
\hline
\end{tabular}

Value are expressed as a mean and standard error of the mean (SEM) or percentage. HOMA-IR, Homeostatic model assessment of insulin resistance; HOMA-B, Homeostatic model assessment of $\beta$-cell function HOMA-S; Homeostatic model assessment of insulin sensitivity TyG index, triglyceride-glucose index hsCRP; high sensitivity $\mathrm{C}$-reactive protein, $\mathrm{HbA}_{1 \mathrm{c}}$ glycated haemoglobin, TG/HDL ratio; triglyceride to high density lipoprotein. $P$ values are for the comparison between "Non-diabetic "and "Diabetic "subjects

against development of insulin resistance and type $2 \mathrm{DM}$ [37]. This supports the notion that a plant-based food pattern with a balanced glycaemic index and load, rich in soluble fiber and phytochemicals, would be effective to reduce risk of dysglycaemia and prediabetes states.
The Mediterranean and DASH diets are relatively rich in fat from vegetable sources (extra-virgin olive oil, tree nuts) and include an abundance of minimally processed plantfoods (vegetables, fruits, whole grains, legumes), moderate fish consumption, low consumption of meat and meat 


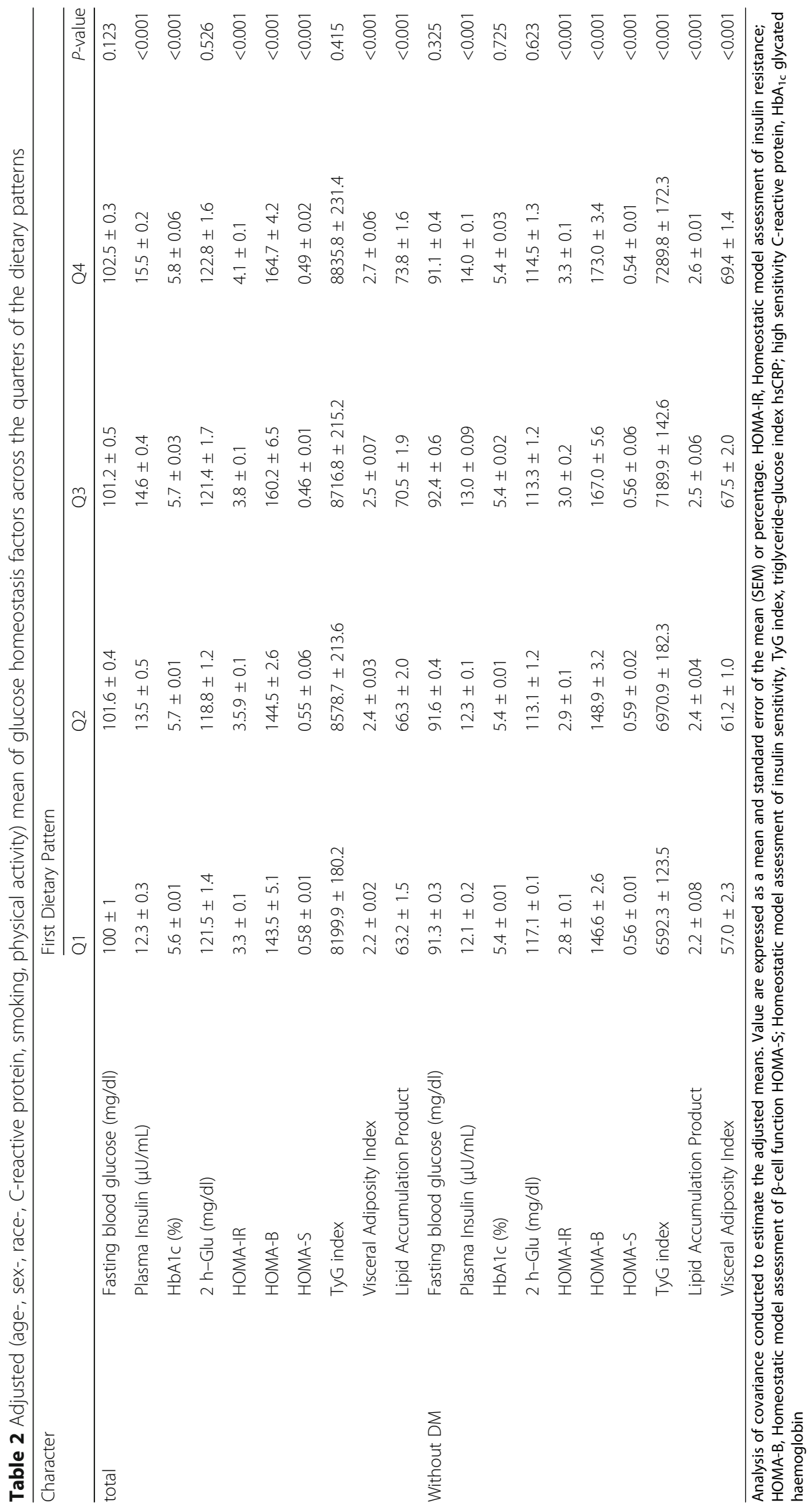




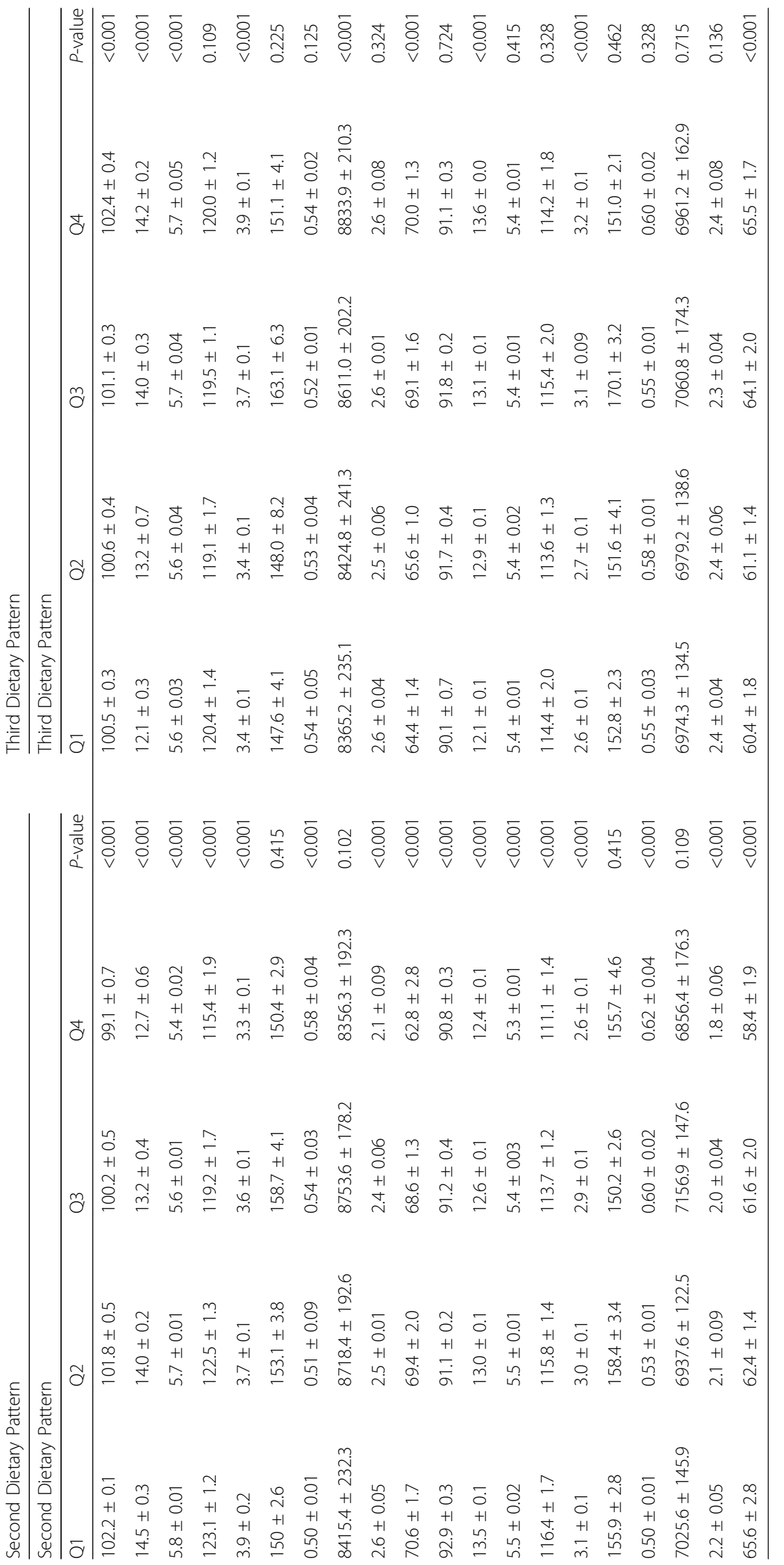


products, and wine in moderation, usually consumed with meals [38]. It has been proposed that their favourable impact could be due to their components [38]. For example, in meta-analysis and systematic review of 17 original research studies (1 clinical trial, 9 prospective and 7 crosssectional) of 136,846 participants, higher adherence to the Mediterranean diet was associated with a $23 \%$ reduced risk of developing type 2 diabetes [39]. There is a plausible biological explanation. The antioxidant profile of the diet may suppress oxidative stress accumulation, which has been reported to mediate the development of insulin resistance and $\beta$-cell dysfunction [40]. In addition, magnesiumrich foods, such as vegetables, nuts and legumes can prevent a magnesium deficiency. It was found that decreased intracellular enzymatic activity, attributed to magnesium deficiency, might favour insulin resistance [41], while extracellular magnesium is also needed to prevent a rise in intracellular calcium concentration, which impairs insulin signalling as well [42]. Another mechanism implicates soluble dietary fiber, particularly those found in cereal [43]. Their beneficial properties could derive from high magnesium concentrations or delayed gastric emptying, which slows down digestion and glucose absorption and reduces plasma insulin levels [44]. Moreover, moderate alcohol consumption has been associated with enhanced insulin sensitivity, possibly through adiponectin or HDL-C [45], whereas resveratrol, a phytophenol primarily found in wine, has also been implicated in improved insulin signalling [45].

Consistent with current findings, in subjects without DM, the Framingham Offspring Study showed that adherence to both the 'refined grains and sweets' and 'soda' dietary patterns compared with the 'dietary pattern high in fruits or reduced fat dairy and whole grains' pattern (Mediterranean diet) were associated with higher $2 \mathrm{~h}$ post-challenge insulin levels [46, 47]. Moreover, confectionaries and soft drinks, may deteriorate the glucose metabolism according to the possibly unfavourable effect of diets high in simple sugars on insulin sensitivity [48].

This study has several strengths. The large sample provided adequate statistical power to evaluate for any significant associations. The selection of the participants was based on random sampling of the general population and therefore the results obtained from nationally representative samples can be extrapolated to the general population. As the data collection was performed on all days of the week throughout the year in NHANES, the potential for selection bias is low $[49,50]$. Moreover, the present study is one of the biggest to investigate derived dietary patterns in relation to relatively comprehensive parameters of glucose and insulin homeostasis in an American population, over time.

The present study has some limitations. Its crosssectional nature does not allow inferences about causality.
Data on glycaemic index and glycaemic load were unavailable, and accordingly, their possible effect could not be explored. In the current study, a one-day $24 \mathrm{~h}$ food record was used, which may not capture long-term diet. Moreover, there is a possibility of over- and underreporting food intake. Analyses in this paper are based on data collected over a 10-year period. It is likely that the diet of American people changed over this period or subsequently, which may to some extent affect the generalizability of the findings.

While the current study findings reinforce the importance of balanced diet, the uncovered links between some of the nutrients and glucose and insulin homeostasis may represent novel metabolic pathways and provide basis for further research. This raises the possibility that glucose and insulin homeostasis could be improved by changes in lifestyle, particularly by diet.

\section{Conclusions}

This study suggests that the dietary pattern enriched with $\mathrm{CHO}$, SFA, total fat and, PUFA, protein, MUFA as well as high-cholesterol-load foods is associated with impaired glucose tolerance. However, the healthy pattern which is high in vitamins, minerals and fiber appear to have favourable effects on insulin sensitivity and glucose tolerance. The principal implications of the study highlight the importance of dietary patterns in the development and prevention of dysglycaemia and impaired insulin homeostasis.

\section{Acknowledgments}

MM was supported by a TWAS and Chinese Academy of Sciences.

Funding

None.

Availability of data and materials

All the data are from public access database.

Authors' contributions

MM, APK contributed to the study concept and design, data analysis and interpretation, and drafting of the manuscript. DPM, PPT, KKR, data analyses and critical revision of the manuscript for important intellectual content. MB, contributed to the design of the study, data analyses and critical revision of the manuscript for important intellectual content, and drafting of the manuscript. All authors read and approved the final manuscript.

Ethics approval and consent to participate

For the data collection and physical examination of the NHANES, informed consent was obtained from all adult participants, and the National Centre for Health Statistics Research Ethics Review Board approved the protocol.

\section{Consent for publication}

For the data collection and physical examination of the NHANES, informed consent (publication) was obtained from all adult participants, and the National Centre for Health Statistics Research Ethics Review Board approved the protocol.

Competing interests

The authors declare that they have no competing interests. 


\section{Publisher's Note}

Springer Nature remains neutral with regard to jurisdictional claims in published maps and institutional affiliations.

\section{Author details \\ "Key State Laboratory of Molecular Developmental Biology, Institute of Genetics and Developmental Biology, Chinese Academy of Sciences, Chaoyang, Beijing, China. ${ }^{2}$ Institute of Genetics and Developmental Biology College, University of Chinese Academy of Science, Beijing, China. ${ }^{3}$ Non-Communicable Disease Research Unit, South African Medical Research Council and University of Cape Town, Cape Town, South Africa. ${ }^{4}$ Department of Clinical Biochemistry, University College London Medical School, University College London (UCL), London, UK. ${ }^{5}$ Department of Preventive Cardiology, CGH Medical Center, Sterling, IL, USA. ${ }^{6}$ Ciccarone Center for the Prevention of Cardiovascular Disease, Johns Hopkins University School of Medicine, Baltimore, MD, USA. ${ }^{7}$ Department of Public Health and Primary Care, University of Cambridge, Cambridge, UK. ${ }^{8}$ Department of Hypertension, Chair of Nephrology and Hypertension, Medical University of Lodz, Lodz, Poland. ${ }^{9}$ Polish Mother's Memorial Hospital Research Institute (PMMHRI), Lodz, Poland. ${ }^{10}$ Cardiovascular Research Centre, University of Zielona-Gora, Zielona-Gora, Poland.}

\section{Received: 27 June 2017 Accepted: 18 September 2017}

Published online: 04 October 2017

\section{References}

1. Alonso LC, Yokoe T, Zhang P, Scott DK, Kim SK, O'donnell CP, et al. Glucose infusion in mice. Diabetes. 2007:56(7):1792-801.

2. Ashcroft FM, Rorsman P. Diabetes mellitus and the $\beta$ cell: the last ten years. Cell. 2012;148(6):1160-71

3. Fung TT, Schulze M, Manson JE, Willett WC, Hu FB. Dietary patterns, meat intake, and the risk of type 2 diabetes in women. Arch Intern Med. 2004, 164(20):2235-40

4. Du T, Yuan G, Zhang M, Zhou X, Sun X, Yu X. Clinical usefulness of lipid ratios, visceral adiposity indicators, and the triglycerides and glucose index as risk markers of insulin resistance. Cardiovasc Diabetol. 2014;13:146.

5. Kang B, Yang Y, Lee EY, Yang HK, Kim HS, Lim SY, et al. Triglycerides/ glucose index is a useful surrogate marker of insulin resistance among adolescents. Int J Obes. 2017:41(5):789-92.

6. He Y, Ma G, Zhai F, Li Y, Hu Y, Feskens EJ, et al. Dietary patterns and glucose tolerance abnormalities in Chinese adults. Diabetes Care. 2009:32(11):1972-6.

7. Williams DE, Prevost AT, Whichelow MJ, Cox BD, Day NE, Wareham NJ. A cross-sectional study of dietary patterns with glucose intolerance and other features of the metabolic syndrome. Br J Nutr. 2000:83(3):257-66.

8. Hu FB. Dietary pattern analysis: a new direction in nutritional epidemiology. Curr Opin Lipidol. 2002;13(1):3-9.

9. Newby P, Tucker KL. Empirically derived eating patterns using factor or cluster analysis: a review. Nutr Rev. 2004;62(5):177-203.

10. Movassagh EZ, Vatanparast H. Current Evidence on the Association of Dietary Patterns and Bone Health: A Scoping Review. Adv Nutr. 2017:8(1):1-16.

11. Zuo H, Shi Z, Yuan B, Dai Y, Pan X, Wu G, et al. Dietary patterns are associated with insulin resistance in Chinese adults without known diabetes. Br J Nutr. 2013:109(09):1662-9.

12. Mazidi M, Kengne AP. Nutrient patterns and their relationship with general and central obesity in US adults. Eur J Clin Investig. 2017. doi:10.1111/eci.12745.

13. Mazidi M, Pennathur S, Afshinnia F. Link of dietary patterns with metabolic syndrome: analysis of the National Health and Nutrition Examination Survey. Nutrition \& diabetes. 2017;7(3):e255

14. Slattery ML. Defining dietary consumption: is the sum greater than its parts? Am J Clin Nutr. 2008;88(1):14-5.

15. Yu R, Woo J, Chan R, Sham A, Ho S, Tso A, et al. Relationship between dietary intake and the development of type 2 diabetes in a Chinese population: the Hong Kong Dietary Survey. Public Health Nutr. 2011;14(7): 1133-41.

16. Oba S, Nanri A, Kurotani K, Goto A, Kato M, Mizoue T, et al. Dietary glycemic index, glycemic load and incidence of type 2 diabetes in Japanese men and women: the Japan Public Health Center-based Prospective Study. Nutr 2013:12(1):165.

17. Montonen J, Knekt P, Harkanen T, Jarvinen R, Heliovaara M, Aromaa A, et al. Dietary patterns and the incidence of type 2 diabetes. Am J Epidemiol. 2005;161(3):219-27.
18. National Center for Health Statistics CfDCaPNHaNESA, https://wwwn.cdc gov/nchs/nhanes/ f. Accessed 25 Jan 2016.

19. Mazidi M, Kengne AP, Mikhailidis DP, Cicro AF, Banach M. Effects of selected dietary constituents on high-sensitivity C-reactive protein levels in U.S. adults. Ann Med. 2017. doi:10.1080/07853890.2017.1325967. Accessed 25 Oct 2016.

20. Mazidi M, Gao HK, Vatanparast H, Kengne AP. Impact of the dietary fatty acid intake on C-reactive protein levels in US adults. Medicine (Baltimore). 2017;96(7):e5736. doi:10.1097/MD.0000000000005736.

21. Mazidi M, Michos ED, Banach M. The association of telomere length and serum 25-hydroxyvitamin D levels in US adults: the National Health and Nutrition Examination Survey. Arch Med Sci. 2017;13(1):61-5.

22. Needham BL, Adler N, Gregorich S, Rehkopf D, Lin J, Blackburn EH, et al. Socioeconomic status, health behavior, and leukocyte telomere length in the National Health and Nutrition Examination Survey, 1999-2002. Social science \& medicine (1982). 2013:85:1-8.

23. Remer T. Influence of nutrition on acid-base balance-metabolic aspects. Eur J Nutr. 2001:40(5):214-20.

24. Musso G, Gambino R, Bo S, Uberti B, Biroli G, Pagano G, et al. Should nonalcoholic fatty liver disease be included in the definition of metabolic syndrome? A cross-sectional comparison with Adult Treatment Panel III criteria in nonobese nondiabetic subjects. Diabetes Care. 2008;31(3):562-8.

25. Simental-Mendia LE, Rodriguez-Moran M, Guerrero-Romero F. The product of fasting glucose and triglycerides as surrogate for identifying insulin resistance in apparently healthy subjects. Metab Syndr Relat Disord. 2008; 6(4):299-304.

26. Report of the Expert Committee on the Diagnosis and Classification of Diabetes Mellitus. Diabetes Care. 1997;20(7):1183-97.

27. Matthews DR, Hosker JP, Rudenski AS, Naylor BA, Treacher DF, Turner RC. Homeostasis model assessment: insulin resistance and beta-cell function from fasting plasma glucose and insulin concentrations in man. Diabetologia. 1985;28(7):412-9.

28. Samouda H, Dutour A, Chaumoitre K, Panuel M, Dutour O, Dadoun F. VAT= TAAT-SAAT: innovative anthropometric model to predict visceral adipose tissue without resort to CT-Scan or DXA. Obesity (Silver Spring). 2013;21(1):E41-50.

29. Amato MC, Giordano C, Galia M, Criscimanna A, Vitabile S, Midiri M, et al. Visceral Adiposity Index: a reliable indicator of visceral fat function associated with cardiometabolic risk. Diabetes Care. 2010;33(4):920-2.

30. Onat A, Avci GS, Barlan MM, Uyarel H, Uzunlar B, Sansoy V. Measures of abdominal obesity assessed for visceral adiposity and relation to coronary risk. Int J Obes Relat Metab Disord. 2004;28(8):1018-25.

31. Statistics. NCfH. Analytic and reporting guidelines. https:/wwwn.cdc.gov/nchs/ nhanes/nhanes0304/nhanesanalyticguidelinesdec2005.pdf.

32. Khayyatzadeh SS, Moohebati M, Mazidi M, Avan A, Tayefi M, Parizadeh SM, et al. Nutrient patterns and their relationship to metabolic syndrome in Iranian adults. Eur J Clin Investig. 2016;46(10):840-52.

33. Stanhope KL. Role of fructose-containing sugars in the epidemics of obesity and metabolic syndrome. Annu Rev Med. 2012:63:329-43.

34. Doostvandi T, Bahadoran Z, Mozaffari-Khosravi H, Mirmiran P, Azizi F. Food intake patterns are associated with the risk of impaired glucose and insulin homeostasis: a prospective approach in the Tehran Lipid and Glucose Study. Public Health Nutr. 2016;19(13):2467-74.

35. Li M, Fan Y, Zhang X, Hou W, Tang Z. Fruit and vegetable intake and risk of type 2 diabetes mellitus: meta-analysis of prospective cohort studies. BM J Open. 2014;4(11):e005497.

36. Schulze MB, Hoffmann K, Manson JE, Willett WC, Meigs JB, Weikert C, et al. Dietary pattern, inflammation, and incidence of type 2 diabetes in women. Am J Clin Nutr. 2005;82(3):675-84.

37. Batis C, Mendez MA, Sotres-Alvarez D, Gordon-Larsen P, Popkin B. Dietary pattern trajectories during 15 years of follow-up and $\mathrm{HbA1c}$, insulin resistance and diabetes prevalence among Chinese adults. J Epidemiol Community Health. 2014;68(8):773-9.

38. Willett WC, Sacks F, Trichopoulou A, Drescher G, Ferro-Luzzi A, Helsing E, et al. Mediterranean diet pyramid: a cultural model for healthy eating. Am J Clin Nutr. 1995:61(6 Suppl):1402s-6s.

39. Koloverou E, Esposito K, Giugliano D, Panagiotakos D. The effect of Mediterranean diet on the development of type 2 diabetes mellitus: a meta-analysis of 10 prospective studies and 136,846 participants. Metabolism. 2014;63(7):903-11.

40. Evans JL, Goldfine ID, Maddux BA, Grodsky GM. Are oxidative stressactivated signaling pathways mediators of insulin resistance and beta-cell dysfunction? Diabetes. 2003;52(1):1-8. 
41. Barbagallo M, Dominguez LJ, Galioto A, Ferlisi A, Cani C, Malfa L, et al. Role of magnesium in insulin action, diabetes and cardio-metabolic syndrome $X$. Mol Asp Med. 2003;24(1-3):39-52.

42. McCarty MF. PKC-mediated modulation of L-type calcium channels may contribute to fat-induced insulin resistance. Med Hypotheses. 2006;66(4): 824-31.

43. Lau C, Faerch K, Glumer C, Tetens I, Pedersen O, Carstensen B, et al. Dietary glycemic index, glycemic load, fiber, simple sugars, and insulin resistance: the Inter99 study. Diabetes Care. 2005;28(6):1397-403.

44. Chandalia M, Garg A, Lutjohann D, von Bergmann K, Grundy SM, Brinkley L. Beneficial effects of high dietary fiber intake in patients with type 2 diabetes mellitus. N Engl J Med. 2000;342(19):1392-8.

45. Bonnet F, Disse E, Laville M, Mari A, Hojlund K, Anderwald CH, et al. Moderate alcohol consumption is associated with improved insulin sensitivity, reduced basal insulin secretion rate and lower fasting glucagon concentration in healthy women. Diabetologia. 2012;55(12):3228-37.

46. Liu E, McKeown NM, Newby P, Meigs JB, Vasan RS, Quatromoni PA, et al. Cross-sectional association of dietary patterns with insulin-resistant phenotypes among adults without diabetes in the Framingham Offspring Study. Br J Nutr. 2009;102(04):576-83.

47. Booth JN 3rd, Colantonio LD, Howard G, Safford MM, Banach M, Reynolds K, et al. Healthy lifestyle factors and incident heart disease and mortality in candidates for primary prevention with statin therapy. Int J Cardiol. 2016; 207:196-202.

48. Daly ME, Vale C, Walker M, Alberti K, Mathers JC. Dietary carbohydrates and insulin sensitivity: a review of the evidence and clinical implications. Am J Clin Nutr. 1997;66(5):1072-85.

49. Tooze JA, Midthune D, Dodd KW, Freedman LS, Krebs-Smith SM, Subar AF, et al. A new statistical method for estimating the usual intake of episodically consumed foods with application to their distribution. J Am Diet Assoc. 2006;106(10):1575-87.

50. Guenther PM, Ding EL, Rimm EB. Alcoholic beverage consumption by adults compared to dietary guidelines: results of the National Health and Nutrition Examination Survey, 2009-2010. J Acad Nutr Diet. 2013;113(4):546-50.

\section{Submit your next manuscript to BioMed Central and we will help you at every step:}

- We accept pre-submission inquiries

- Our selector tool helps you to find the most relevant journal

- We provide round the clock customer support

- Convenient online submission

- Thorough peer review

- Inclusion in PubMed and all major indexing services

- Maximum visibility for your research

Submit your manuscript at wuw biomedcentral.com/submit

) Biomed Central 